\title{
Using magnetic nanoparticles to improve flux pinning in $\mathrm{YBa}_{2} \mathrm{Cu}_{3} \mathrm{O}_{\mathrm{x}}$ films
}

\author{
Masih Mojarrad \\ Department of Physics \\ University of Oslo \\ Oslo, Norway \\ masih.mojarrad@fys.uio.no
}

\author{
V. S. Dang \\ Nano and Energy Center, VNU Hanoi \\ University of Science, 334 Nguyen Trai, \\ Thanh Xuan, \\ Hanoi, Vietnam \\ dangvson@gmail.com
}

\author{
Jouan Hamid \\ Department of Physics \\ University of Oslo \\ Oslo, Norway \\ j.h.hamid@fys.uio.no
}

A. Crisan

National Institute for Materials Physics

Bucharest, 405A Atomistilor Str.,

077125

Magurele, Romania

adrian.crisan@infim.ro

\author{
Ana Lucía Campaña \\ Department of Physics \\ University of Oslo \\ Oslo, Norway \\ a.l.c.perilla@fys.uio.no
}

\author{
Pavlo Mikheenko \\ Department of Physics \\ University of Oslo \\ Oslo, Norway \\ pavlo.mikheenko@fys.uio.no
}

\begin{abstract}
Superconductors application can lead to significant economic benefits, especially in combination with use of liquid hydrogen, which is becoming an important part of the renewable energy economy. While many traditional superconductors cannot operate in liquid hydrogen, new materials, like high-temperature superconductors and $\mathrm{MgB}_{2}$ perfectly suit this purpose. $\mathrm{YBa}_{2} \mathrm{Cu}_{3} \mathrm{O}_{\mathrm{x}}$ is one of the most used high-temperature superconductors. It can operate even in liquid nitrogen, at the temperature of $77.3 \mathrm{~K}$, but has a strong advantage of enhanced critical current density at the boiling temperature of liquid hydrogen of $20 \mathrm{~K}$. A disadvantage of this material is the absence of natural c-axis pinning centers defining its critical current density. A usual way to solve this problem is the introduction of artificial pinning centers in the form of nanoparticles. The nanoparticles, however, reduce the volume of the superconductor and can lead to the formation of high-angle grain boundaries detrimental for the critical current. Here we explore an approach of depositing magnetic nanoparticles on the surface of superconducting films, which neither reduce the volume of the superconductor nor create high-angle grain boundaries. The additional pinning by these nanoparticles is studied by recording magneto-optical images of the films.
\end{abstract}

Keywords-superconductivity, magneto-optical imaging, nanoparticle, Yttrium Barium Copper Oxide, magnetic pinning centers.

\section{INTRODUCTION}

Superconducting industry strongly matured in recent years becoming ready for challenges such as fusion reaction [1]. Another challenge would be the introduction of superconductors in suggested Norwegian liquid hydrogen value chain. In this chain, most of the sea fleet on the west coast of Norway: high-speed boats, ferries, and ships serving oil platforms will use clean liquid hydrogen as a fuel strongly reducing $\mathrm{CO}_{2}$ emissions in the atmosphere. Since the proper coolant is already there, a smart design of the vessels would allow use of superconducting motors, generators, current limiters, energy storage units, and other key elements necessary to run the chain smoothly, safely, efficiently, and economically affordable.

Among the materials, which could be used for the Norwegian liquid hydrogen value chain, are high- temperature superconductors, primarily $\mathrm{YBa}_{2} \mathrm{Cu}_{3} \mathrm{O}_{\mathrm{x}}$ (YBCO), and $\mathrm{MgB}_{2}$. YBCO is not easy in preparation, as it requires nearly perfect crystallinity combined with a very dense array of artificial pinning centers, but the proper technologies are already developed, see, for example, [2]. Artificial pinning centers trap magnetic vortices increasing critical current density in the superconductors [3, 4]. For the fusion purposes, the incorporation of $\mathrm{Y}_{2} \mathrm{O}_{3}$ nanoparticles into YBCO appeared to be sufficient [1]. For other applications, the introduction of columnar defects, like, for example, described in [5] might be preferable. Nano inclusions such $\mathrm{WO}_{3}$ [6], $\mathrm{TiO}_{2}$ [2], $\mathrm{BiFeO}_{3}$ [7] and $\mathrm{BaTiO}_{3}$ [8] were found efficient in enhancing critical current density. A search for other possibilities to improve pinning should not be neglected too.

One of the interesting ideas is to use magnetic nanoparticles to improve pinning in YBCO. It was already explored in the range of publications, incorporating the nanoparticles by laser deposition on top or bottom of the superconducting layer [9] or producing them by alternating laser ablation of YBCO and the material of nanoparticles $[10,11]$. In [9], the film with nanoparticles deposited on the top of YBCO showed larger critical current density than in those with magnetic nanoparticles on the substrate or superconducting film without nanoparticles.

Following these observations, our approach is to apply magnetic Fe nanoparticles on top of already prepared YBCO films from a commercial solution. To make it more challenging, the YBCO films with columnar defects were chosen for this experiment, with already enhanced pinning properties comparable to the plain superconducting films. The advantage of suggested method is the ease of adding nanoparticles after the preparation of YBCO. To observe their effect, the magneto-optical technique was employed, which allows to directly visualize penetration of magnetic flux into the superconductor.

\section{EXPERIMENTAL METHODS}

The YBCO films were pulse laser deposited on $\mathrm{SrTiO}_{3}$ substrates having orientation (100) using an excimer $\mathrm{KrF}$ 
$248 \mathrm{~nm}$ laser with the duration of the pulses of $30 \mathrm{~ns}$. The pulse repetition rate was $5 \mathrm{~Hz}$. The substrates, to which the films were deposited, were kept at the temperature of 780 ${ }^{\circ} \mathrm{C}$. To achieve the columnar growth of YBCO [5], the Au nanoparticles were deposited on the substrate prior to the deposition of the superconductor by a number of laser pulses on the Au target. The different number of laser pulses on Au target results in different size of the nanoparticles and different coverage of the substrate. When YBCO is ablated, it dissolves in the molten nanoparticles and deposits epitaxially on the substrate under them forming YBCO nano-rods. Two different YBCO samples, S1 and S2, were prepared with the number of laser pulses to Au targets of 10 and 15, respectively. Both YBCO films are about $800 \mathrm{~nm}$ thick.

A $5 \mathrm{mg} / \mathrm{mL}$ solution of single core magnetic Fe oxide nanoparticles of $10 \mathrm{~nm}$ average diameter was purchased from a commercial supplier (Ocean Nanotech, San Diego, CA, USA). The nanoparticles are coated with an amino functionalization, so they can be stably monodispersed in aqueous solutions. For this application, the Fe nanoparticles were dissolved in distilled water to reach a concentration of $5 \mu \mathrm{g} / \mathrm{mL}$. A drop of solution was spread on the top of the films and allowed to dry naturally. For S1, the procedure was repeated twice, investigating magnetic flux penetration into the samples at temperatures below the critical temperature of YBCO after each deposition. Although there were concerns that water could be harmful to YBCO, it appeared that it was not influencing negatively the films. After an application of nanoparticles, the surface of one of the films (S2) was examined by Atomic Force Microscopy (AFM) using a JPK Nano-Wizard 4 with a 4.0 pyramidaltipped silicon cantilever, PPP-MFMR-10 (Nanosensors, Switzerland), to confirm magnetism and estimate the roughness of the surface. The tip with the magnetic coating of cobalt has an approximated sharpness of $50 \mathrm{~nm}$. The study was carried in $\mathrm{AC}$ mode at a resonance frequency of the cantilever of $74.6 \mathrm{kHz}$.

The penetration of magnetic flux into the films has been monitored by magneto-optical imaging (MOI) [12, 13]. This technique uses Faraday's rotation effect in specially prepared indicator films, which modify the polarization of light in the presence of magnetic field. It is a fast technique with simple interpretation of the images, which could be used on the industrial scale [14].

\section{RESULTS AND DISCUSSION}

Fig. 1 shows magneto-optical images for the film S1 before $(a, b)$ and after $(c, d)$ the deposition of 10-nm Fe nanoparticles. The images show the penetration of magnetic flux (bright contrast) at the application of a magnetic field of $85 \mathrm{mT}$ after zero-field cooling to $70 \mathrm{~K}$.

The image a) has been recorded about three years after the deposition of the film and 10 years before recording other images. While similarities between the details of the flux penetration are obvious in all MOI maps, several internal defects appeared in the film with time, which provided additional penetration of the magnetic flux inside the sample according to the scenario described in [15].

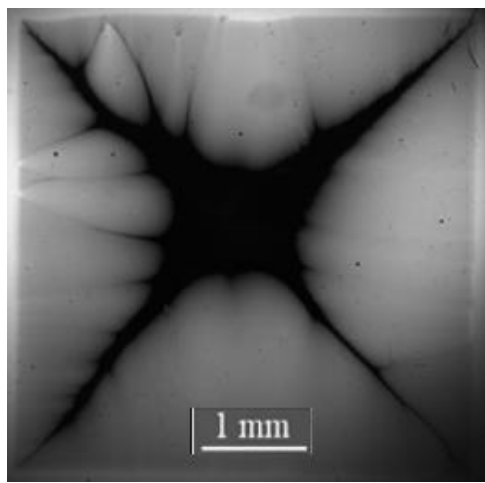

a)

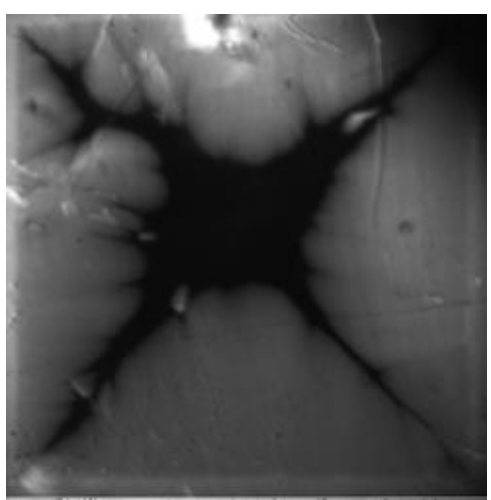

b)

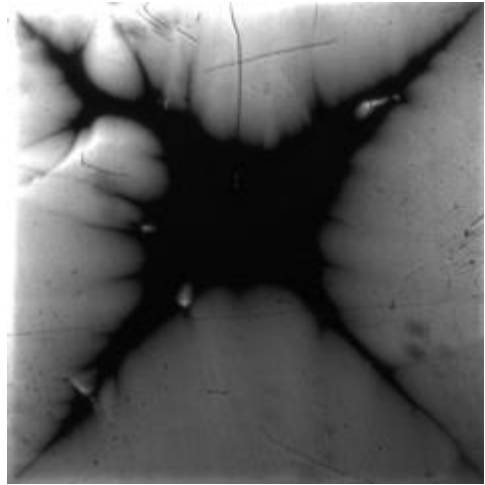

c)

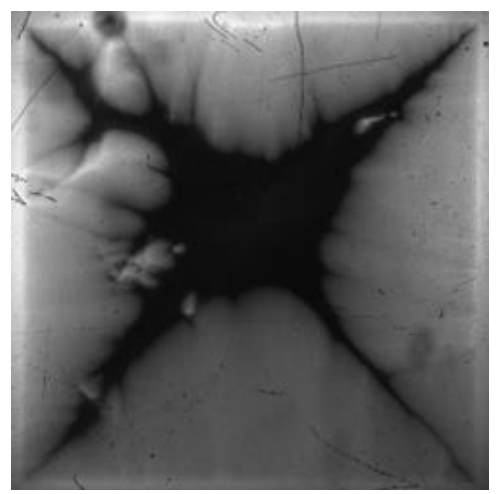

d)

Fig.1 Magneto-optical images for the film S1 before (a,b) and after (c,d) the deposition of 10-nm Fe nanoparticles. The image a) has been recorded about 10 years before recording other images. In c), 10-nm magnetic nanoparticles were deposited to the film, and in d), these nanoparticles were deposited twice.

In spite of additional local penetration, the black area in the images, which is free from the magnetic flux, is bigger in b) than in a). This is a known effect of the improvement with time over several years of critical current density in YBCO columnar films, which was reported in [16]. Comparing to this long scale, images in b) to d) were 
recorded at approximately the same time and no changes in the size of the black area would be expected. The observed difference between these images comes then from the fact that in b) the surface of the film was clean, but in c) $10-n m$ magnetic nanoparticles were deposited on it. In d), these nanoparticles were deposited twice.

The most striking effect is that after the first deposition of Fe nanoparticles, the size of the black area significantly increased (compare c) and b)), i.e. magnetic flux pinning induced by these nanoparticles became considerably stronger. The second deposition (d) reduces black area, but it still remains bigger than in $b$ ).

Similar imaging at the application of a magnetic field of $85 \mathrm{mT}$ after zero-field cooling to $70 \mathrm{~K}$ has been done on the sample S2, which contained a larger number of $\mathrm{Au}$ nanoparticles on the substrate than in $\mathrm{S} 1$. The images for S2 are shown in Fig.2.
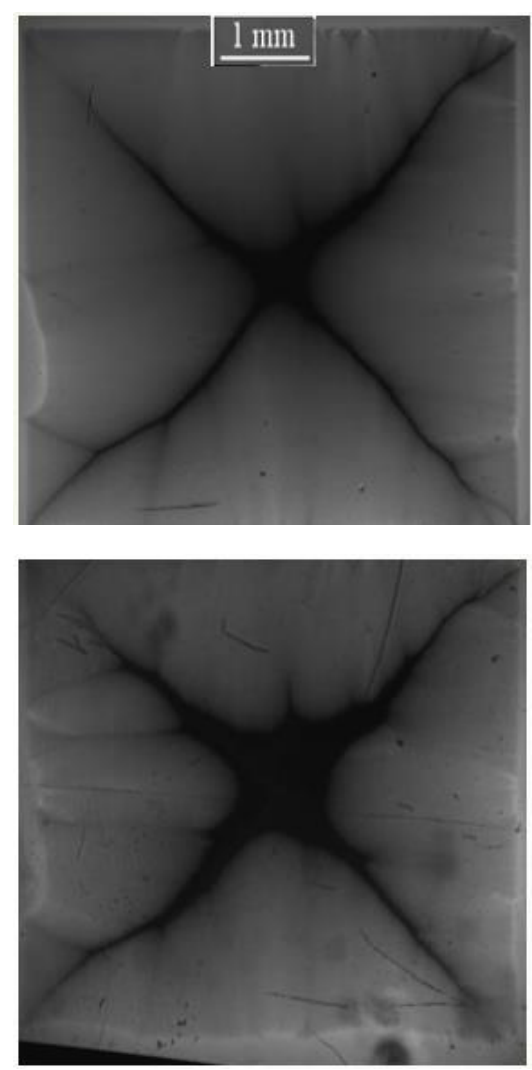

b)

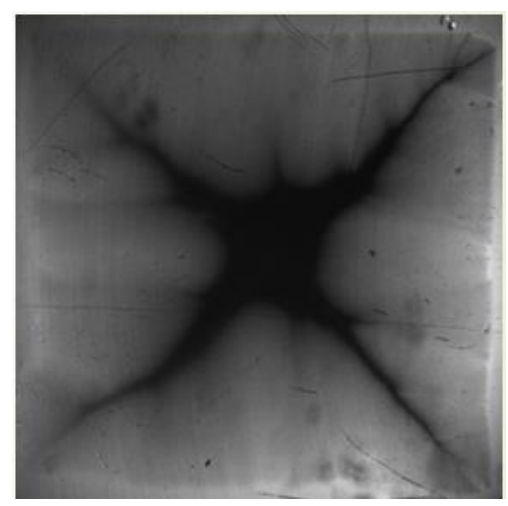

c)

Fig.2 Magneto-optical images for the film S2 before (a,b) and after (c) the deposition of 10-nm Fe nanoparticles. The image a) has been recorded about 10 years before recording b) and c). In c), 10-nm magnetic nanoparticles were covering the film.
Again, the image a) in Fig.2 has been recorded about 10 years before recording $b$ ) and c). The long-time effect on critical current density is stronger in this sample than in S1, since there is a bigger change in the size of the dark area (compare a) and b) for both S1 and S2). There is also an improvement in pinning after applying a layer of magnetic nanoparticles (c)

To obtain quantitative information, the images in Figs. 1,2 were analyzed with software ImageJ calculating the flux filling ratio, i.e. the ratio of the flux-free black area to the flux-filled bright area. The software divides the images into two colors using adjustable settings. Ideally, to find this ratio the whole sample should be analyzed, but due to limitations of the instrument, some images do not have appropriate quality in the corners. Therefore, ImageJ cannot properly recognize the contrast there, which leads to considerable error in the analyses. To avoid such an error, the analyzed areas were restricted in size to identical squares in the middle part of the samples.

The analyses in these areas provides reasonably accurate and trustable data. Another issue to deal with was that the images were taken with variable contrast, which means that they have different thresholds for dividing the pictures into two colors. To solve this problem, an optimization of the contrast has been done using software of ImageJ.

The final result of the analysis is shown by black squares with corresponding error bars in Fig.3 for the samples S1 (a) and S2 (b).

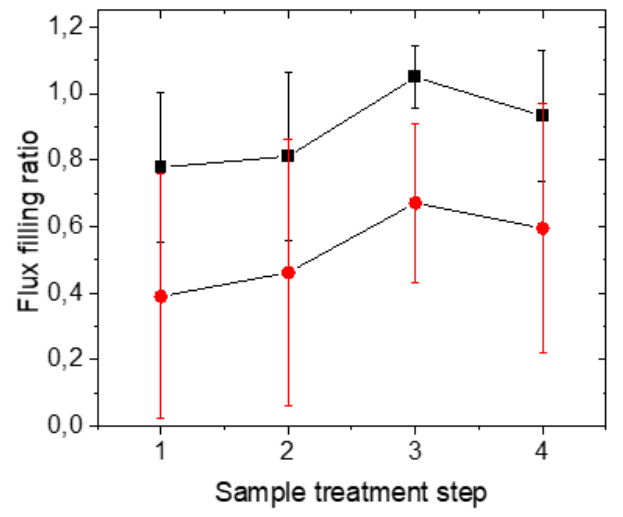

a)

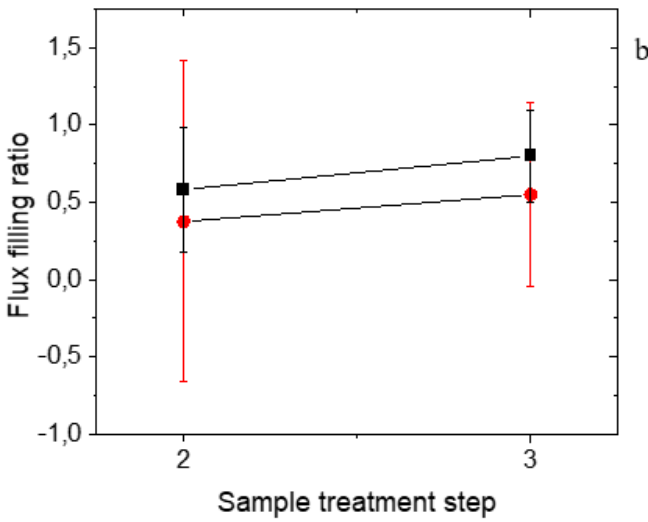

Fig.3 Flux filling ratio for different sample treatment steps for the sample S1 (a) and S2 (b). The sample treatment steps 1 to 4 in a) correspond to images a) to d) in Fig. 1, and the sample treatment steps 2 and 3 in b) correspond to images b) and c) in Fig. 2. The red circles in a) and $b$ ) give additional data for MOI images recorded with the application of a higher than for black squares magnetic field. 
The sample treatment steps 1 to 4 in Fig. 3a correspond to images a) to d) in Fig. 1, and the sample treatment steps 2 and 3 in Fig. 3 b correspond to images b) and c) in Fig. 2. The red circles give additional data for MOI images recorded with the application of the magnetic field of 113 $\mathrm{mT}$ in $\mathrm{S} 1$ and $98 \mathrm{mT}$ in $\mathrm{S} 2$ after the zero-field cooling to 70 K.

Both the positive effect of long-term aging (steps 1 and 2) and the improvement of flux pinning after the application of magnetic nanoparticles (steps 2 and 3) are seen in Fig. 3a. However, there is a deterioration in pinning properties after the exposure to a larger concentration of magnetic nanoparticles (steps 3 and 4) for sample S1 (Fig. 3a). The improvement of flux pinning after the application of magnetic nanoparticles (steps 2 and 3) is also seen in S2 (Fig. 3b) at different applied magnetic fields.

According to the plots in Fig 3, after the application of one layer of ferromagnetic nanoparticles, the improvement in flux filling ratio is $29-45 \%$ for the sample $\mathrm{S} 1$ and $37-46 \%$ for the sample S2. This is rather a considerable effect of magnetic nanoparticles that could be used in practical applications as a final step in the preparation of superconducting wires or tapes

To have better insight into the nature of magnetic pinning, the surface of S2 was examined by the magnetic force microscopy after the deposition of magnetic nanoparticles. The corresponding images are shown in Fig. 4. Plot a) in Fig. 4 shows the surface topography of the sample. The outgrowths of the in-plane sub-micrometer size and the height of up to $60 \mathrm{~nm}$ are seen in it. The borders of the outgrowths are outlined in the amplitude of the probe oscillations map b) treated with the edge detection technique. This technique seems to reveal some of the nanocolumns. In c), the magnetic signal (dark color) is detected by the phase shift map [17]. The magnetic nanoparticles are accumulated preferentially along the borders of the outgrowths. The height of the outgrowths is a small fraction of the thickness of the film, which is about $800 \mathrm{~nm}$. Still, they may considerably contribute to the surface pinning [18]. From this point of view, the action of the magnetic nanoparticles could be considered as an enhancement of the surface pinning.

\section{CONCLUSION}

An enhancement of magnetic flux pinning in columnargrown superconducting YBCO films has been achieved by covering their surface with magnetic Fe nanoparticles. The magnetic force microscopy reveals that nanoparticles are mainly concentrated at the borders of the outgrowths on the surface, which are reaching the height of up to $60 \mathrm{~nm}$.

The effect could be considered as an enhancement of the surface pinning. The excessive density of nanoparticles leads to the suppression of the pinning. The deposition of the nanoparticles could be recommended as a final step in the preparation of practical superconductors.
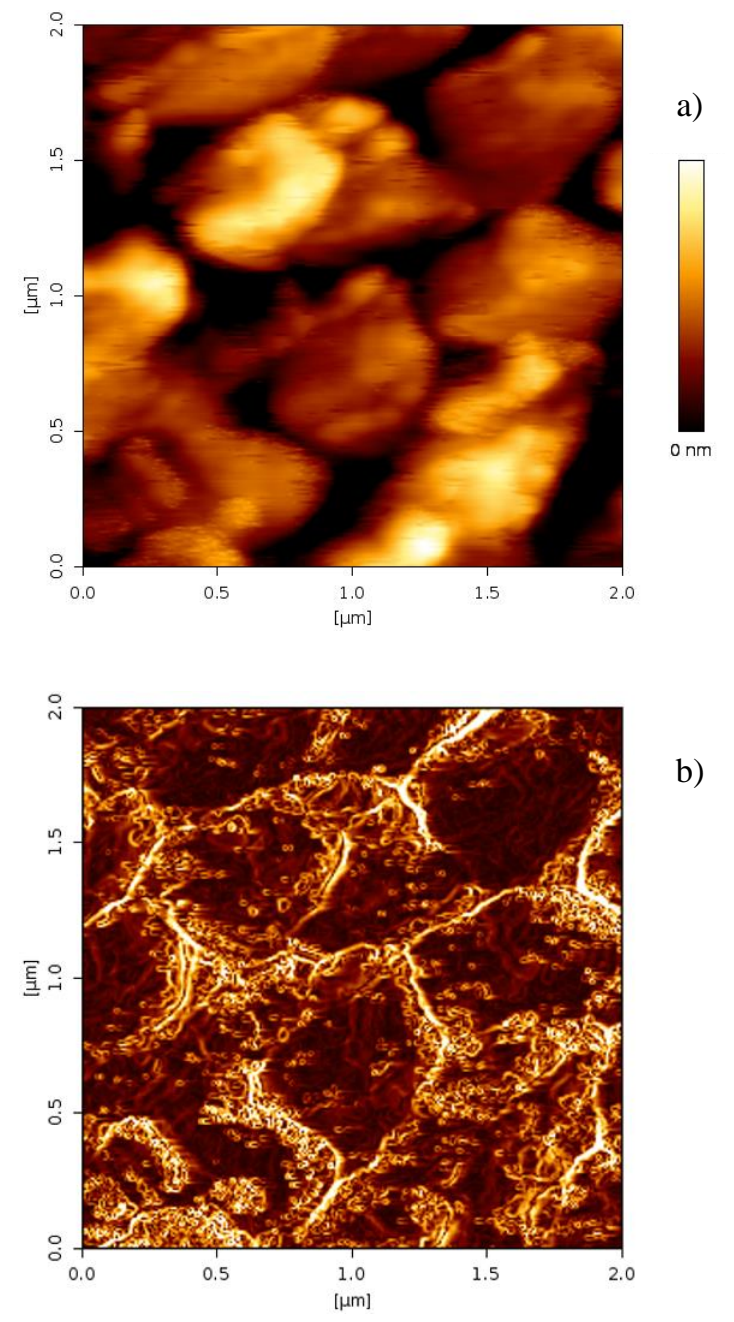

b)

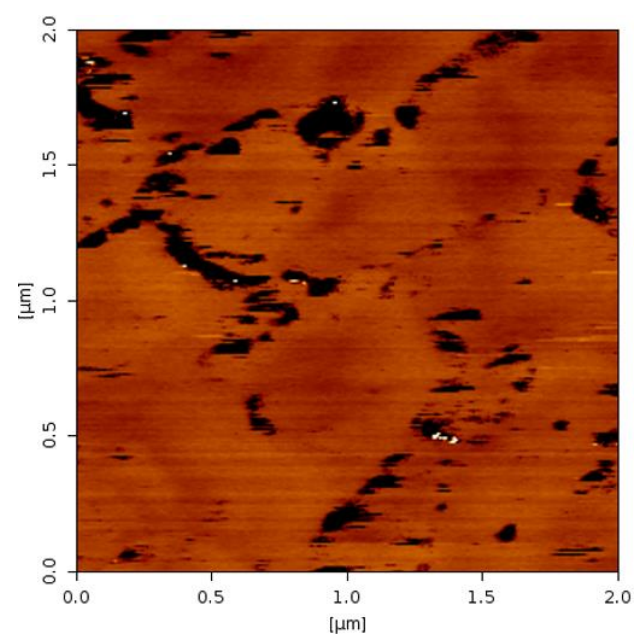

c)

Fig.4 Magnetic force microscopy images of the sample S2 with deposited magnetic nanoparticles. a) Topography of the surface revealing the outgrowths of the height of up to $60 \mathrm{~nm}$. b) Amplitude map for the probe oscillations treated with the edge detection technique to reveal nanocolumns and the edges of the outgrowths. c) Magnetic signal (dark color) detected in the phase shift map.

\section{ACKNOWLEDGMENT}

We would like to thank Assoc. Prof. Irep Gözen and her group for providing magnetic nanoparticles and Prof. Geir Helgesen for his scientific support. 


\section{REFERENCES}

[1] A. Molodyk et al., "Development and large volume production of extremely high current density $\mathrm{YBa}_{2} \mathrm{Cu}_{3} \mathrm{O}_{7}$ superconducting wires for fusion," Scientific reports, vol. 11, no. 1, pp. 1-11, 2021.

[2] E. Hannachi, Y. Slimani, F. B. Azzouz, and A. Ekicibil, "Higher intra-granular and inter-granular performances of YBCO superconductor with $\mathrm{TiO}_{2}$ nano-sized particles addition," Ceramics International, vol. 44, no. 15, pp. 18836-18843, 2018.

[3] F. Antončík, O. Jankovský, T. Hlásek, and V. Bartůněk, "Nanosized pinning centers in the rare earth-barium-copper-oxide thin-film superconductors," Nanomaterials, vol. 10, no. 8, p. 1429, 2020.

[4] A. Ushakov, I. Karpov, A. Lepeshev, and M. Petrov, "Enhancing of magnetic flux pinning in $\mathrm{YBa}_{2} \mathrm{Cu}_{3} \mathrm{O}_{7-\mathrm{x}} / \mathrm{CuO}$ granular composites," Journal of Applied Physics, vol. 118, no. 2, p. 023907, 2015.

[5] A. Crisan, V.-S. Dang, and P. Mikheenko, "Nano-engineered pinning centres in YBCO superconducting films," Physica C: Superconductivity and its Applications, vol. 533, pp. 118-132, 2017.

[6] Y. Slimani et al., "Improvement of flux pinning ability by tungsten oxide nanoparticles added in $\mathrm{YBa}_{2} \mathrm{Cu}_{3} \mathrm{O}_{\mathrm{y}}$ superconductor," Ceramics International, vol. 45, no. 6, pp. 6828-6835, 2019.

[7] G.-Z. Li, S.-Y. Wang, J.-W. Li, and W.-M. Yang, "Introducing multisource pinning centers into $\mathrm{Y}-\mathrm{Ba}-\mathrm{Cu}-\mathrm{O}$ superconductor through addition of $\mathrm{BiFeO}_{3}$ nano-particles," Scripta Materialia, vol. 132, pp. 22-24, 2017.

[8] P. Rejith, S. Vidya, and J. Thomas, "Effect of addition of $\mathrm{BaTiO}_{3}$ nano particles on the electrical transport properties of YBCO superconductor," in IOP Conference Series: Materials Science and Engineering, 2015, vol. 73, no. 1: IOP Publishing, p. 012146.

[9] J. Wang, C.-F. Tsai, Z. Bi, D. G. Naugle, and H. Wang, "Microstructural and Pinning Properties of $\mathrm{YBa}_{2} \mathrm{Cu}_{3} \mathrm{O}_{7-\delta}$ Thin Films Doped With Magnetic Nanoparticles," IEEE transactions on applied superconductivity, vol. 19, no. 3, pp. 3503-3506, 2009.
[10] M. A. Sebastian, N. A. Pierce, I. Maartense, G. Kozlowski, and T. J. Haugan, "Effect of Nano-Size Magnetic Additions on Low Temperature Flux Pinning of Y-Ba-Cu-O Thin Films," IEEE Transactions on Applied Superconductivity, vol. 31, no. 5, pp. 1-7, 2021.

[11] C.-F. Tsai, Y. Zhu, L. Chen, and H. Wang, "Flux pinning properties in YBCO thin films with self-aligned magnetic nanoparticles," IEEE transactions on applied superconductivity, vol. 21, no. 3, pp. 27492752, 2010 .

[12] T. H. Johansen and D. Shantsev, Magneto-optical imaging. Dordrecht: Springer Science \& Business Media, 2004.

[13] P. Mikheenko, V. Yurchenko, D. Cardwell, Y. Shi, and T. Johansen, "Magneto-optical imaging of superconductors for liquid hydrogen applications," Journal of superconductivity and novel magnetism, vol. 26, no. 5, pp. 1499-1502, 2013.

[14] Y. Deng, Y. Cheng, L. Xuan, and Z. Zeng, "Principles of magnetooptic imaging and its applications," Integrated Imaging and Vision Techniques for Industrial Inspection, pp. 483-536, 2015.

[15] P. Mikheenko, "Excitation of Vortex-Antivortex Pairs in Thin Superconducting Films and Superlattices," in Advances in Thin Films, Nanostructured Materials, and Coatings: Springer, 2019, pp. 277-285.

[16] P. Mikheenko et al., "Magneto-optical imaging of columnar YBCO films," Physics Procedia, vol. 36, pp. 623-628, 2012.

[17] A. L. Campaña, N. Joudeh, H. Høyer, A. Røyne, D. Linke, and P. Mikheenko, "Probing Van Der Waals and Magnetic Forces in Bacteria with Magnetic Nanoparticles," in 2020 IEEE 10th International Conference Nanomaterials: Applications \& Properties (NAP), 2020: IEEE, pp. 01NSSA03-1-01NSSA03-5.

[18] A. Kosse et al., "Geometrical surface vortex pinning in superconducting films," Journal of Experimental and Theoretical Physics Letters, vol. 78, no. 6, pp. 379-383, 2003. 